\title{
Electrical properties of wood colonized by Gloeophyllum trabeum
}

\author{
Grant T. Kirker, Amy B. Bishell, Samuel L. Zelinka ${ }^{1}$ \\ US Forest Service, Forest Products Laboratory | 1 Gifford Pinchot Dr. |Madison, WI \\ 53726, USA
}

\section{Abstract}

This paper examines how wood decay fungi affect the electrical resistance (resistivity) of wood to determine whether an electrical resistance probe could be deployed as a remote sensor in a wall to detect wood decay. Electrical resistance measurements were taken on wood blocks exposed to Gloeophyllum trabeum in a standard soil bottle tests (AWPA E10) as a function of time. To understand how and why resistance changed with time, results were compared against resistance measurements taken in sterilized, un-inoculated wood at high moisture contents; concentrations of certain mineral ions in the wood were also measured. Results indicate that most of the changes in electrical resistance in the soil bottle test could be described by changes in moisture content alone. When the moisture effects were removed, decay fungi decreased the resistance at small amounts of weight loss but increased the resistance at high weight loss. The results presented herein help to explain the mechanism by which empirical, electrical resistance nondestructive testing devices work and also the limitations on using resistance measurements to detect wood decay.

Keywords: wood decay, nondestructive evaluation, electrical resistance measurements, wood moisture content, brown rot fungus, ionic conduction

\section{Introduction}

Wood decay fungi can cause catastrophic strength loss in buildings, bridges, and other structures but are often hard to detect from a visual examination because the surface may appear sound while the interior is decayed (Clausen and Kartal, 2003; Wilcox, 1978). Because of this, a host of nondestructive evaluation methods have been used to examine wood for decay (De Groot et al., 1998; Ross and Pellerin, 1994). Although much recent work has focused on ultrasound and/or stress wave evaluation (Ross et al., 1998), earlier work by Shigo showed that in some cases electrical resistance measurements could be used to detect decay (Shortle and Smith, 1987). Shigo and Shigo (1974) developed the eponymous Shigometer, a device used to detect wood decay in standing trees or utility poles using electrical resistance measurements that was based upon anecdotal evidence by Lin (1967). The Shigometer was a lightweight ohmmeter that used a pulsed direct current through twisted copper wires to measure changes in resistance along an internal transect of a presumably decayed tree or pole. Shigo

\footnotetext{
${ }^{1}$ Corresponding author szelinka@fs.fed.us
} 
and Shigo (1974) found that the measured resistance would drop when the probe moved from sound to decayed wood and developed empirical criteria to detect decay. Because the decay criteria were based upon a calibration developed from destructive evaluation, the Shigometer could be used only in situations that mimicked the calibration conditions. The Shigometer could not be used on frozen wood, north-facing aspects of trees, or most importantly "overly wet wood" (Morris and Dickinson, 1984; Paysen et al., 1992). The Shigometer was used extensively in the 1980 s for detection of decay in utility poles and standing trees (Shigo and Shigo, 1974).

Although the empirical Shigometer can be used in certain situations, for electrical measurements to be used more broadly to detect decay, we must understand the mechanism for charge conduction in wood and how wood decay affects this mechanism. Wood is an ionic conductor, whose conductivity $(\sigma)$ can be described by

$$
\sigma=n z \mu(\text { Eq 1) }
$$

where $n$ is the number of charge carriers, $z$ is their charge, and $\mu$ is their mobility, which is related to their diffusion coefficient (Glass and Zelinka, 2010; Lin, 1965). The conductivity of wood is strongly dependent upon moisture content, increasing by over 10 orders of magnitude from oven dry to full saturation (Glass and Zelinka, 2010). Zelinka et al. (2008) showed that below fiber saturation, the increase in conductivity can be attributed to an increase in mobility $(\mu)$ described by percolation theory. The Shigometer detected decay by measuring resistance, which can be calculated from the reciprocal of conductivity (resistivity, $\rho$ ) and a geometrical factor.

Physically, there are several possible explanations for how decay fungi could increase the conductivity (and therefore lower the resistance) of wood. One possibility is that decay fungi increase the number of charge carriers $(n)$ or increase the mobility of the charge carriers in the wood $(\mu)$. A second possibility is that in wood with decay, current flows through the fungus and the fungus is more conducting than the wood cell wall. Finally, it is possible that decay fungi do not affect resistance at all but are strongly correlated with another variable that affects resistance (wood moisture content). In this final case, decay fungi are not affecting the resistivity of the wood but the decayed wood has a lower resistance than sound wood because it has more moisture than the surrounding wood.

At least some experimental evidence indicates that part of the change in conductivity may be caused by an increase in the number of charge carriers. Connolly and Jellison (1997) demonstrated that $G$.

trabeum, a brown rot fungus, was able to translocate cations to and from decayed blocks of red spruce sapwood but did not measure changes in electrical conductivity. Furthermore, it is unclear what role these translocated ions play in the changes in conductivity of decayed wood; perhaps the increase in conductivity caused by ions is dwarfed by changes in mobility or wood moisture content. In short, it is unclear why decayed wood has a low measured resistance, as there has been no systematic investigation of how decay affects both mobility and ion concentration and comparison of the data to sound wood at the same moisture content.

This work explores the mechanism by which wood decay fungi affect the conductivity of wood, with an eye towards developing an improved wood decay detection device that may be used in buildings. The 
resistance of small wooden blocks was measured after exposure to $G$. trabeum in a soil bottle over time between 2 and 8 weeks. G. trabeum was chosen as it is a model test fungus for wood degradation that has been used in numerous past studies and there are literature data on how this fungus translocates ions from the soil (Schilling and Jellison, 2007). The measurements are compared against a "calibration curve," where the resistance of the blocks was measured as a function of moisture content, to account for increased conductance with moisture. These measurements allow a clear, systematic, investigation of how and why decay fungi affect resistance measurements in wood.

\section{Methods and Materials}

\subsection{Wood specimens}

Stainless steel screws were placed into the end grain of southern pine (Pinus spp.) blocks (19 mm $\times 19$ $\mathrm{mm} \times 76.2 \mathrm{~mm}$ ) (Figure 1), which allowed repeatable electrical connection to be made to the blocks for resistance measurements. The average weight of the screws, $1.88 \mathrm{~g}$, was obtained by weighing 50 screws at once to maximize the accuracy of the balance. This average weight was used to correct for the weight of the screws when calculating wood moisture content and percentage weight loss caused by decay.

\subsection{Electrical measurements and the effect of moisture on the resistance of non-decayed wood} Before starting the decay test, "calibration curve" data were collected by fixing the wood moisture content of the blocks and measuring resistance. Although the relationship between wood moisture content and resistivity is well characterized in the hygroscopic region (below fiber saturation), much less is known about the overhygroscopic region (above fiber saturation but below complete water saturation) (Brischke and Lampen, 2014; Brischke et al., 2008; Fredriksson et al., 2013; James, 1963, 1988; Stamm, 1929). Because wood decay is most active in the overhygroscopic region (Griffin, 1977), this calibration curve is essential for understanding how wood decay changes electrical properties. However, these measurements are challenging because the overhygroscopic region represents a region on the wood water phase diagram where two phases are in equilibrium (Zelinka et al., 2016b). In a twophase equilibrium, the chemical potential of each component is the same in both phases, but the relative amounts of the phases can change on the total composition (Callister, 2003). For the woodwater system, this means that the overhygroscopic region contains a range of possible moisture contents at a given chemical potential (Zelinka et al., 2016b). Likewise, adding liquid water to blocks results in an uneven moisture gradient and erroneous electrical measurements. The measurements are also challenging because fungal activity is favored at these moisture contents, and mold contamination is a possibility.

Several approaches have been taken to measure the electrical properties in the overhygroscopic region. Fredriksson et al. (2013) used a pressure plate apparatus to equilibrate wood at very high water potentials $\left(<a_{w}<\right)$. Brischke $(2014 ; 2008)$ fully saturated wood and stored it under cold conditions (to prevent biological growth) while letting the sample dry to $50 \%$ moisture content. We developed a method to obtain a calibration curve at many different moisture contents in the overhygroscopic region without fungal contamination. Oven-dry wood blocks were placed in $50 \mathrm{ml}$ conical centrifuge tubes that 
could be autoclaved, and various water volumes were delivered with a pipette to the containers to obtain the desired moisture content. For high moisture contents, the water could not be absorbed as fast as it left the pipette, but the water was fully absorbed by the end of the sterilization process. The lids were then screwed tightly on the containers prior to steam sterilization at $121^{\circ} \mathrm{C}$ for 20 minutes in a Steris Amsco Lab 250 autoclave (Mentor, $\mathrm{OH}$ ). Following the autoclave cycle, the containers were stored in the laboratory for several weeks. No mass change was observable during this time, suggesting that the seal was good. The blocks were weighed and their resistances were measured to determine the moisture dependence of resistance above the fiber saturation point.

Resistances were measured with an apparatus built in-house specifically designed to overcome the polarization effects frequently found in wood and give repeatable measurements. The apparatus, fully described in work by Boardman et al. (2012), applied $10 \mathrm{~V}$ across a 200-k $\Omega$ precision resistor in series with the specimen. The resistance of the wood was then calculated by measuring voltage drop across the precision resistor. The technique prevented polarization by switching the polarity of the bias voltage every second and averaging a group of four measurements. Three consecutive measurements were made on each block, so the reported value represents an average of 12 measurements. Consecutive readings were stable, suggesting that the measured resistances were not affected by polarization. In previous work (Boardman et al., 2012; Zelinka et al., 2016a) the uncertainties in the electrical measurements were found to be less than $4 \%$ (typically less than $2 \%$ ) when measuring resistances within a factor of 1000 from the shunt resistor (in this case between $200 \Omega$ to $200 \mathrm{M} \Omega$ ).

\subsection{Decay Test}

Pre-test oven-dry weights of the blocks were recorded, and the blocks were sterilized by autoclave. Soil bottles were set up according to the AWPA standard E10 (Anon, 2012). Southern pine feeder strips $40 \mathrm{~mm} \times 30 \mathrm{~mm} \times 3 \mathrm{~mm}$ were pre-drilled with a central hole to accommodate the stainless steel screws on either end of the test blocks. The strips were placed on water-soaked soil in glass bottles, then sterilized by autoclave. Once cool, feeder strips were inoculated with two 10-mm plugs of mycelium from the leading edge of $G$. trabeum cultures on malt extract agar. The fungus was allowed to establish on the feeder strip for three weeks, and then the sterilized blocks were placed into prepared sterilized soil bottles and stored in a room maintained at $27^{\circ} \mathrm{C}$ and $70 \% \mathrm{RH}$. All blocks were placed so that the end-grain of the block was exposed to the mycelium-covered feeder strip; this is also the direction along which electrical measurements were taken.

Specimens were removed from the soil bottles at weekly intervals from 2 to 8 weeks after being placed in the soil bottle. Specimens were weighed and their electrical resistance measured. Specimens were then oven-dried, and the percentage weight loss caused by decay was calculated from the difference in initial and final oven-dry weights. Moisture content $\left(g_{\text {water }} / g_{\text {dry material }}\right)$ was calculated from the difference in mass immediately after being removed from the decay chamber and post-test oven-dry weight.

To see if the living mycelium were acting as an additional electrical path and contributing to the decrease in resistance, additional experiments were run on the decayed blocks. In these experiments, the decayed blocks were oven dried to kill the fungus, and then rewetted to the approximately the same moisture content they were at the end of the decay test. However, we were unable to rewet the blocks 
to exactly the same moisture content. Because small changes in the wood moisture content have such a large influence on the electrical resistance, these data contained too much noise and uncertainty to make any observations (data not shown).

\section{4 lon concentration}

Concentration of ions in the wood was examined using inductively coupled plasma atomic emission spectroscopy (ICP-AES) (Anon, 2007a, b). Wood samples weighing approximately $0.5 \mathrm{~g}$ were digested by placing them into $5 \mathrm{~mL}$ of nitric acid (70\% concentration). The solution was heated with microwave radiation to $150^{\circ} \mathrm{C}$ for 20 min under pressure and then brought up to $50 \mathrm{~mL}$ volume with water. An initial, semi-quantitative survey showed differences in the amount of calcium, manganese, and magnesium between the decayed and non-decayed specimens. Quantitative analysis was performed on these three ions for blocks that had been decayed for 4 and 8 weeks.

\section{Results and Discussion}

Resistance of undecayed blocks as a function of moisture content (calibration curve) is plotted in Figure 2; data from Fredriksson et al. (2013) are included for reference. Previously Zelinka et al. (2008)

observed that electrical resistivity of wood exhibits a percolation model with power-law behavior above the percolation threshold, which they found to be $16 \%$ moisture content (MC). The calibration data are consistent with a power law model, and the solid line represents the resistance $R$ as a function of the moisture content $\mathrm{m}$, given below,

$$
R(\text { in } \mathrm{k} \Omega)=0.80 m^{-5.9}(\text { Eq 2.) }
$$

which was found to be the best-fit line to a power law model $\left(R^{2}=0.84\right)$.

Electrical resistance of the decayed blocks is shown in Figure 3 (as a function of time in Figure 3a). Resistance appears to decrease from weeks 2 to 4 , after which resistance levels off. When examined this way, the data suggest that measured resistance, and thus wood resistivity, is affected by the decay fungi at least in the early stages of decay.

A relationship between the initial stages of wood decay and resistance also appears in Figure $3 \mathrm{~b}$, which plots resistance as a function of weight loss caused by decay. These are the same data shown in Figure $3 a$ but are plotted in terms of weight loss instead of experiment time. A drop in resistance is clearly evident until approximately $10 \%$ weight loss, at which point resistance does not change with increasing weight loss but instead remains constant.

Although Figures 3a and $b$ show that resistivity of wood decreases in the initial stages of the soil bottle test and suggest possible causation between decay and a drop in resistance, a more nuanced picture emerges from Figures 3c. In a soil bottle test, wood moisture content is not held constant but is allowed to equilibrate with moisture in the soil. When the same data from Figures $2 a$ and $b$ are plotted as a function of moisture content at the end of the test, clearly moisture also affects measured resistance. In fact, the data have the same shape as the calibration curve taken in sound blocks (Eq 2, overlaid). 
From Figures 3a-c, measured resistance clearly depends upon moisture content and potentially also the amount of fungal decay. Figure $3 d$, a plot of "moisture-normalized resistance" as a function of percentage weight loss, separates the effect of moisture from the effect of decay. Moisture-normalized resistance $\left(R_{\text {norm }}\right)$ was calculated by normalizing the measured resistance to the resistance at $30 \% \mathrm{MC}$. $30 \% \mathrm{MC}$ was chosen because it was both the mean and median moisture content of all of the decayed blocks. Therefore, normalizing the resistance to $30 \% \mathrm{MC}$ caused half of the resistances to be increased and half to be decreased.

The normalization was applied by

$$
R_{\text {norm }}=R_{\text {meas }}^{x} \frac{f(0.3)}{f(x)} \text { (Eq. 3) }
$$

where $R_{\text {meas }}^{X}$ is the measured resistance of the block at a moisture content of $x$ and $f(x)$ is the power-law fit to the calibration curve (Eq. 2). By plotting $R_{\text {norm }}$ instead of $R_{\text {meas }}^{X}$, we are able to examine the effect of decay on resistance (resistivity) independent of moisture content. Resistance of the sound blocks at $30 \% \mathrm{MC}$ is shown as a solid line in Figure $3 \mathrm{~d}$; measurements below this line indicate that resistance is lower than it would be if there had been no decay. Figure $3 \mathrm{~d}$ shows a clear trend between $R_{\text {norm }}$ and the amount of decay. As the percentage weight loss increases, $R_{\text {norm }}$ initially decreases, plateaus at a minimum, and then rises with increasing weight loss, eventually having a higher resistance than undecayed blocks at $25 \%$ weight loss. These data show that in the initial stages of decay, resistance is lower than would otherwise be expected in sound wood, but as percentage weight loss further increases, resistance is actually higher than in sound wood.

Results from the ICP-AES experiments are shown in Figure 4. Ions are apparently being translocated from the soil in the soil bottle tests into the wood. Total concentrations of $\mathrm{Ca}, \mathrm{Mn}$, and $\mathrm{Mg}$ were less than $0.6 \mathrm{mg} \mathrm{g}^{-1}$ at the beginning of the test and increased to $1.8 \mathrm{mg} \mathrm{g}^{-1}$ at 4 weeks. This ion translocation increases the number of charge carriers in the wood ( $n$ in Eq. 1). According to Eq. 1, this tripling of the ion concentration should reduce measured resistance to $33 \%$ of the original resistance if there were no changes in the mobility of the ions. In fact, average resistance of the wood decreased from $1600 \mathrm{k} \Omega$ to $350 \mathrm{k} \Omega$ ( $21 \%$ of the original) from weeks 2 to 4 in the decay test. In short, the amount that resistance decreased was more than could be explained by ion translocation alone.

One aspect of the study was to examine whether electrical resistance sensors could be used to detect decay in buildings or bridges. Although resistance decreased dramatically with time in the soil bottle test, most of this decrease could be attributed to the fact that the sample moisture content was increasing during this same time. In the beginning stages of decay, resistance was indeed lower than would otherwise be expected for wood at the same moisture content; however, once the percentage weight loss caused by decay became greater than $25 \%$, resistance was actually greater than it would be in sound blocks. Practically, this would make it difficult to detect decay in buildings because in the beginning stages of decay, resistance is lower than in sound wood, but as the decay increases, it is higher than in sound wood; therefore interpretation of the resistance data would be very difficult. 
Despite the attempt to create a somewhat uniform moisture distribution in the controls, it is possible that these samples had moisture gradients. Furthermore, it is likely that the decayed samples contained moisture gradients. It is known that moisture gradients influence resistance based electrical measurements (Tiitta and Olkkonen, 2002). The presence of moisture gradients may have influenced the shape of the calibration curve as well as the decayed wood blocks. For the calibration curve, the resistance at a given moisture content was found to be lower than the fit of Fredriksson et al. (2013) who conditioned the blocks with a pressure plate and would assumedly have a more even distribution of moisture. This suggests that there were pathways within the wood structure with a higher moisture content (and thus lower resistance) that were causing the average resistance to be lower than if the moisture was uniformly distributed. The influence of moisture gradients on the resistance of the decayed blocks cannot be determined. In practice however, moisture gradients are sure to exist in environments where decay are likely to grow because in these environments the wood is wetted by liquid water.

Another practical difficulty in detecting decay through resistance measurements is that changes in resistance caused by decay are much smaller than changes in resistance caused by wood moisture content. Measured resistances in sound wood dropped from $45 \mathrm{M} \Omega$ at $16 \% \mathrm{MC}$ to less than $100 \mathrm{k} \Omega$ at $48 \% \mathrm{MC}-\mathrm{a} 50,000 \%$ reduction in the resistance. However, changes in $R_{\mathrm{norm}}$ caused by decay were at most $1 \mathrm{M} \Omega$ on a resistance on the order of $1 \mathrm{M} \Omega$. Separating out the effect of a $1 \mathrm{M} \Omega$ change in the resistance of wood from changes caused by increasing moisture is virtually impossible and also would require knowing the exact wood moisture content through a method other than electrical measurements to compare against a calibration. In short, using electrical measurements to detect decay suffers from technical difficulties resulting from the small magnitude in the change of resistance as well as theoretical difficulties as the decay does not cause a monotonic change in resistance.

In light of these results separating the effects of moisture, ion translocation, and fungal decay on electrical resistance, it makes sense to discuss the success of the empirical Shigometer. The Shigometer measured resistance as a function of probe depth in the wood surface and was used to detect hidden pockets of decay in the center of large beams or utility poles. When there was no decay, resistance remained constant (within $50 \mathrm{k} \Omega$ ) as the probe was placed deeper into the wood. However, in poles with a decay pocket, resistance would decrease as the probe was moved from the (sound) outer surface of the pole to a decayed area in the center of the pole. Shigo and Shigo (1974) reported an average difference of $250 \mathrm{k} \Omega$ between sound and decayed wood.

The Shigometer was effective at detecting decay for two main reasons: first, it relied on a change in resistance to detect decay (rather than an absolute resistance), and the second is that decay is highly correlated with wood moisture content. Because the Shigometer measured resistance as a function of probe depth, at least some of the measurements were taken in sound wood. This allowed a comparison between the sound wood at the surface and potentially decayed wood in the center. Therefore, the absolute magnitude of the resistance was not important, but only changes in the resistance between a control (near surface measurements) and the potentially decayed wood. A change in relative resistance would therefore be a good indicator that something is different (either decay or a change in wood 
moisture content) in the center of the wood. Second, wood in the vicinity of fungal decay would have higher moisture content because the fungus translocates moisture (Griffin, 1977). This will cause a higher moisture content (and thus lower resistance) in a decay pocket in the center of the wood. Because moisture and decay are correlated, both will result in a drop in resistance relative to sound wood.

Unfortunately, the mechanisms by which the Shigometer worked are not easily deployable as a sensor for decay. Although a resistance probe incorporated into a wall could alert someone to the fact that the wall is getting wet, the data in this paper suggest it would be nearly impossible to tell if it is both wet and decayed or merely wet because changes in resistance caused by decay are much smaller than those caused by changes in moisture content. Furthermore, relative differences between sensors may simply indicate a different moisture distribution within the wall rather than a decayed section of sheathing because likely wetting events in buildings (such as wind-driven rain) are highly non-uniform.

\section{Conclusions}

This paper examined how wood decay affects electrical resistance (resistivity) of wood. Resistance measurements were taken in wood blocks that were pulled from soil bottle decay tests at various times. When compared to the amount of time in the soil bottle test or the amount of weight loss caused by decay, there was a correlation with lower resistances as the decay increased; however, moisture content was also increasing at the same time. When resistances were normalized by comparing them to moisture dependence of sound blocks, the effect of decay on resistance was orders of magnitude less than changes caused by moisture differences between samples. From these data the following conclusions can be drawn:

- Resistance of wood decreases during the first stages of a soil bottle test when the initial stages of decay are occurring. However, during this time moisture content of the wood is also increasing. The majority of this decrease in resistance is caused by the increase in moisture content.

- When a "moisture-normalized" resistance is examined, the beginning stages of decay decrease resistance. However, above $30 \%$ weight loss, resistance is higher than would otherwise be expected for sound wood at the same moisture content.

- In all cases, decay fungi affected the moisture-normalized resistance only by at most $1 \mathrm{M} \Omega$ from a starting resistance of $1 \mathrm{M} \Omega$.

- The Shigometer was an effective tool for detecting decay in large wooden members because it examined relative differences in measured resistance in a large block of wood, where at least some of the wood was undecayed and therefore had a built-in control for comparison.

\section{Acknowledgements}

SLZ acknowledges funding from the Presidential Early Career Award for Scientists and Engineers (PECASE). Research was funded by the Forest Products Laboratory of the USDA Forest Service. The 
authors acknowledge the contributions of Kolby Hirth, who performed the ICP-AES measurements. This paper was written and prepared by U.S. Government employees on official time and therefore is in the public domain and not subject to copyright in the United States.

\section{References}

Anon, 2007a. AWPA A7: Standard for Wet Ashing Procedures for Preparing Wood for Chemical Analysis. American Wood Protection Association, Granbury, TX.

Anon, 2007b. AWPA A21: Standard Method for the Analysis of Wood and Wood Treating Solutions by Inductively Coupled Plasma Emission Spectrometry. American Wood Protection Association, Granbury, TX.

Anon, 2012. AWPA E-10: Standard Method for Testing of Wood Preservatives by Laboratory Soil-block Cultures. American Wood Protection Association, Selma, AL.

Boardman, C., Glass, S.V., Carll, C.G., 2012. Moisture meter calibrations for untreated and ACQ-treated southern yellow pine lumber and plywood. Journal of Testing and Evaluation 40, 1.

Brischke, C., Lampen, S.C., 2014. Resistance based moisture content measurements on native, modified and preservative treated wood. European Journal of Wood and Wood Products 72, 289-292.

Brischke, C., Rapp, A.O., Bayerbach, R., 2008. Measurement system for long-term recording of wood moisture content with internal conductively glued electrodes. Building and Environment 43, 1566-1574.

Callister, W.D., 2003. Materials Science and Engineering: An Introduction. Sixth Edition. John Wiley \& Sons, New York.

Clausen, C., Kartal, S., 2003. Accelerated detection of brown-rot decay: Comparison of soil block test, chemical analysis, mechanical properties, and immunodetection. Forest Products Journal 53, 9094.

Connolly, J.H., Jellison, J., 1997. Two-way translocation of cations by the brown rot fungus Gloeophyllum trabeum. International biodeterioration \& biodegradation 39, 181-188.

De Groot, R.C., Ross, R., Nelson, W., 1998. Non-destructive assessment of wood decay and termite attack in southern pine sapwood. Wood Protection 3, 25-34.

Fredriksson, M., Wadsö, L., Johansson, P., 2013. Small resistive wood moisture sensors: a method for moisture content determination in wood structures. European Journal of Wood and Wood Products 71, 515-524.

Glass, S.V., Zelinka, S.L., 2010. Moisture Relations and Physical Properties of Wood, In: Ross, R.J. (Ed.), Wood Handbook. Wood as an Engineering Material. U.S. Department of Agriculture, Forest Service, Forest Products Laboratory.

Griffin, D., 1977. Water potential and wood-decay fungi. Annual Review of Phytopathology 15, 319-329.

James, W.L., 1963. Electric Moisture Meters For Wood. U.S. Forest Service Research Note FPL-08. US Forest Service Forest Products Laboratory, Madison, WI.

James, W.L., 1988. Electric Moisture Meters For Wood. Forest Products Laboratory General Technical Report FPL-GTR-6. US Forest Service Forest Products Laboratory, Madison, WI.

Lin, R.T., 1965. A study of electrical conduction in wood. Ph.D. Dissertation., State College of Forestry at Syracuse University, Syracuse. New York.

Lin, R.T., 1967. Review of the dielectric properties of wood and cellulose. Forest Products Journal 17, 6166.

Morris, P.I., Dickinson, D.J., 1984. The effect of moisture content on the electrical resistance of timber as detected by a pulsed current resistance meter (Shigometer). Proceedings of the International 
Research Group on Wood Protection, IRG-WP 84-2212. International Research Group on Wood Protection, Stockholm, Sweden.

Paysen, T., Narog, M., Zavala, M., 1992. Monitoring electrical resistance in Canyon Live Oak using a Shigometer, Symposium on Ecology and Management of Oak and Associated Woodlands: Perspectives in the Southwestern United States and New Mexico, Symposium conducted at the meeting of US Department of Agriculture, Sierra Vista USA.

Ross, R.J., Brashaw, B.K., Pellerin, R.F., 1998. Nondestructive evaluation of wood. Forest Products Journal 48, 14.

Ross, R.J., Pellerin, R.F., 1994. Nondestructive testing for assessing wood members in structures. Gen. Tech. Rep. FPL-GTR-70 USDA, Department of Agriculture, Forest Service, Madison, WI.

Schilling, J.S., Jellison, J., 2007. Extraction and translocation of calcium from gypsum during wood biodegradation by oxalate-producing fungi. International biodeterioration \& biodegradation 60 , 8-15.

Shigo, A.L., Shigo, A., 1974. Detection of discoloration and decay in living trees and utility poles. Forest Service, US Department of Agriculture, Northeastern Forest Experiment Station.

Shortle, W.C., Smith, K.T., 1987. Electrical properites and rate of decay in Spruce and Fir Wood. Phytopathology 77, 811-814.

Stamm, A.J., 1929. The fiber-saturation point of wood as obtained from electrical conductivity measurements. Industrial and Engineering Chemistry, Analytical Edition 1, 94-97.

Tiitta, M., Olkkonen, H., 2002. Electrical impedance spectroscopy device for measurement of moisture gradients in wood. Review of Scientific Instruments 73, 3093-3100.

Wilcox, W.W., 1978. Review of literature on the effects of early stages of decay on wood strength. Wood and fiber 9, 252-257.

Zelinka, S., Glass, S., Stone, D., 2008. A Percolation Model for Electrical Conduction in Wood with Implications for Wood-Water Relations. Wood and Fiber Science 40, 544-552.

Zelinka, S., L., Passarini, L., Colon Quintana, J.L., Glass, S.V., Jakes, J.E., Wiedenhoeft, A.C., 2016a. Cell wall domain and moisture content influence southern pine electrical conductivity. Wood and Fiber Science 48, 54-61.

Zelinka, S.L., Glass, S.V., Jakes, J.E., Stone, D.S., 2016b. A solution thermodynamics definition of the fiber saturation point and the derivation of a wood-water phase (state) diagram. Wood Science and Technology 50, 443-462. 


\section{Figure Captions}

Figure 1: Wood specimens used in the decay test after 3 weeks of decay with the screws attached.

Figure 2: Resistance of sound (undecayed) blocks as a function of wood moisture content. The blocks were conditioned by adding water and allowing the water to redistribute in sealed, sterile tubes.

Figure 3: Clockwise from top left: (a) Resistance of the decayed blocks as a function of time in the decay chamber, (b) as a function of the weight loss caused by decay, (c) as a function of the moisture content at the end of the decay test. (d) The moisture-adjusted resistance as a function of decay; note this does not decrease as the amount of decay increases.

Figure 4: Results of the Inductively Coupled Plasma-Atomic Emission Spectroscopy (ICP-AES) quantification of bulk calcium, magnesium, and manganese at two time points during the decay test. 

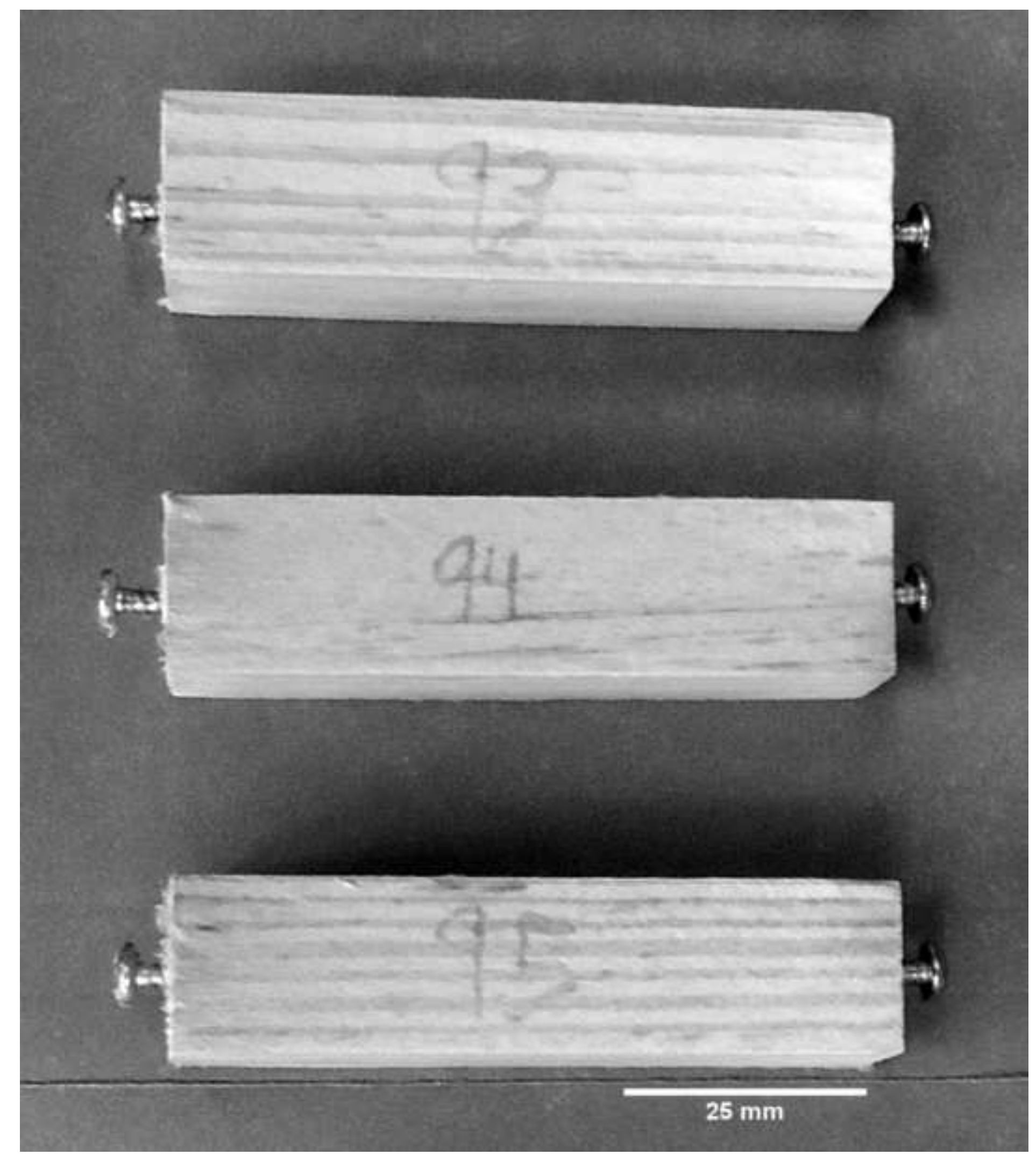

\section{$25 \mathrm{~mm}$}

Figure 1

1

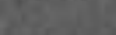

Con

(2)

(2)
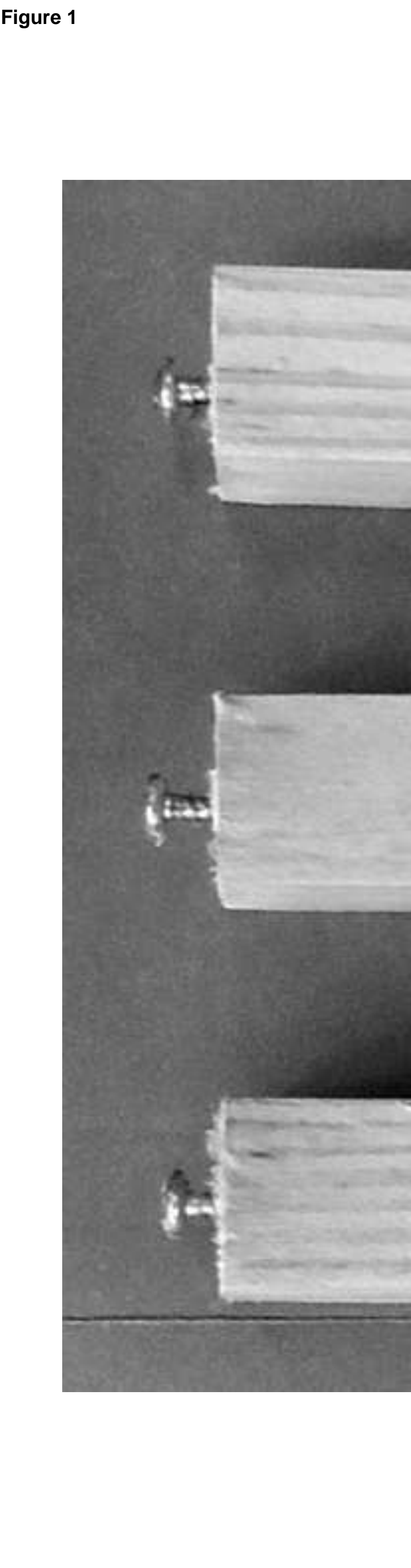


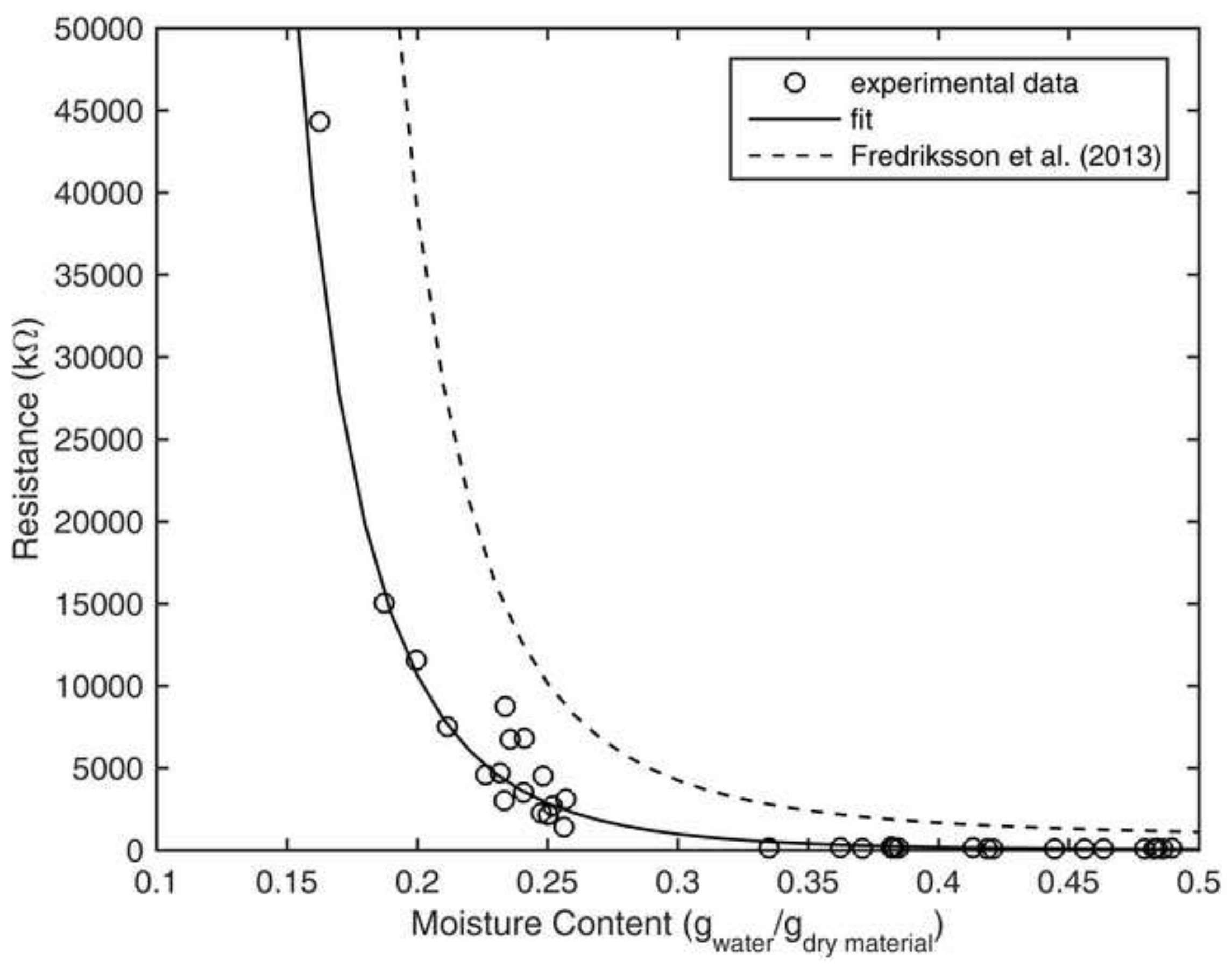



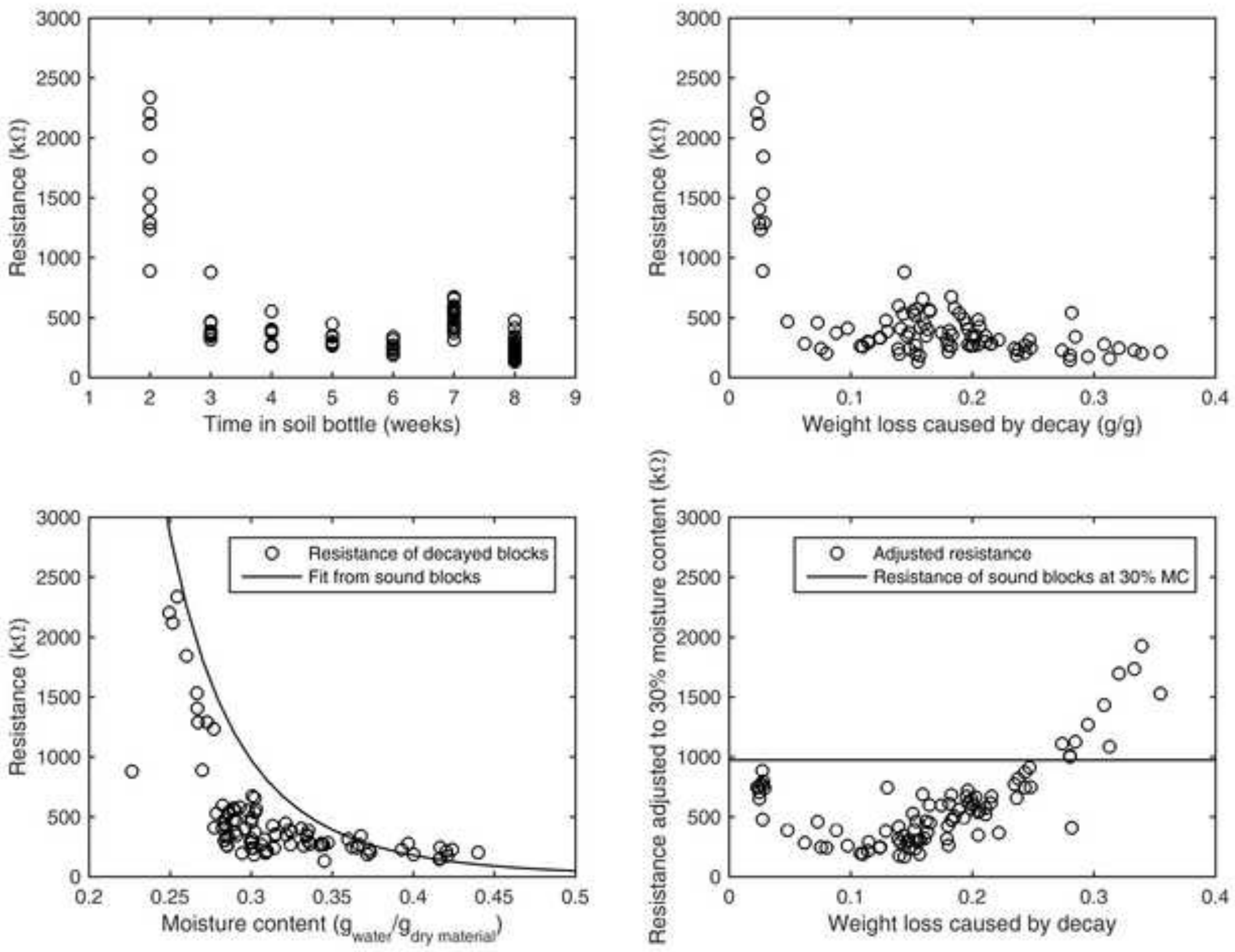


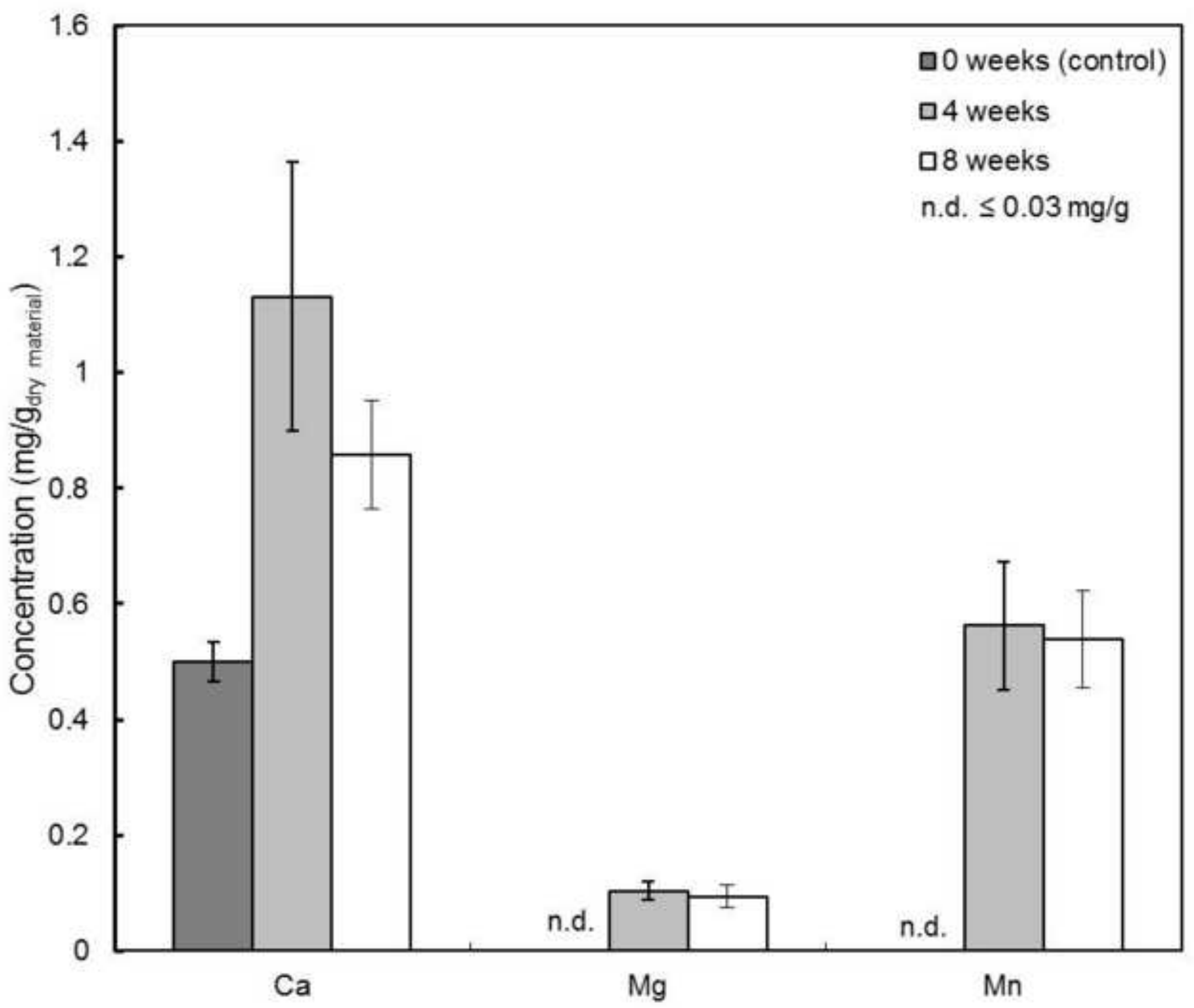

